\title{
Investigating magnetic field dose effects in small animals: a Monte Carlo study
}

\author{
Ashley Erin Rubinstein ${ }^{1,4}$, Michele Guindani ${ }^{3}$, John D Hazle², Laurence E Court ${ }^{1,2}$ \\ ${ }^{1}$ Departments of Radiation Physics, The University of Texas MD Anderson Cancer Center, Houston, TX, USA. \\ ${ }^{2}$ Departments of Imaging Physics, The University of Texas MD Anderson Cancer Center, Houston, TX, USA. \\ ${ }^{3}$ Departments of Biostatistics, The University of Texas MD Anderson Cancer Center, Houston, TX, USA. \\ ${ }^{4}$ Graduate School of Biomedical Sciences, The University of Texas Health Science Center, Houston, TX, USA.
}

Received March 19, 2014; Published Online April 08, 2014

\begin{abstract}
[Presented at the Young Investigator's Symposium at the 2014 Annual Meeting of
Southwest Chapter of American Association of Physicists in Medicine (AAPM) in San Antonio, Texas, USA]
\end{abstract}

\section{Conference Proceeding}

\begin{abstract}
Purpose: In MRI-linac treatments, radiation dose distributions are affected by magnetic fields, especially at high-density/low-density interfaces. Radiobiological consequences of magnetic field dose effects are presently unknown and preclinical studies are desirable. This study investigates the optimal combination of beam energy and magnetic field strength needed for preclinical murine studies.

Methods: The Monte Carlo code MCNP6 was used to simulate the effects of a magnetic field when irradiating a mouse lung phantom with a $1.0 \mathrm{~cm} \times 1.0 \mathrm{~cm}$ photon beam. Magnetic field dose effects were examined using various beam energies (225 kVp, $662 \mathrm{keV}$ [Cs-137], and 1.25MeV [Co-60]) and magnetic field strengths $(0.75 \mathrm{~T}, 1.5 \mathrm{~T}$, and $3 \mathrm{~T})$. The resulting dose distributions were compared to Monte Carlo results for humans with various field sizes and patient geometries using a $6 \mathrm{MV} / 1.5 \mathrm{~T}$ MRI-linac.
\end{abstract}

Results: In human simulations, the addition of a $1.5 \mathrm{~T}$ magnetic field causes an average dose increase of $49 \%$ (range: $36 \%-60 \%)$ to lung at the soft tissue-lung interface and an average dose decrease of $30 \%$ (range: $25 \%$ - 36\%) at the lung-soft tissue interface. In mouse simulations, no magnetic field dose effects were seen with the $225 \mathrm{kVp}$ beam. The

Presenting author: Ashley Erin Rubinstein; Departments of Radiation Physics, The University of Texas MD Anderson Cancer Center, Houston, TX, USA.

Cite this article as:

Rubinstein A, Guindani M, Hazle JD, Court LE. Investigating magnetic field dose effects in small animals: a Monte Carlo study. Int J Cancer Ther Oncol 2014; 2(2):020233.

DOI: $10.14319 /$ ijcto.0202.33 dose increase for the Cs-137 beam was $12 \%$, 33\%, and $49 \%$ for $0.75 \mathrm{~T}, 1.5 \mathrm{~T}$, and $3.0 \mathrm{~T}$ magnetic fields, respectively while the dose decrease was $7 \%, 23 \%$, and $33 \%$. For the Co-60 beam the dose increase was $14 \%, 45 \%$, and $41 \%$, and the dose decrease was $18 \%, 35 \%$, and $35 \%$.

Conclusion: The magnetic field dose effects observed in mouse phantoms using a Co-60 beam with $1.5 \mathrm{~T}$ or $3 \mathrm{~T}$ fields or a Cs-137 beam with a 3T field fall within the range seen in humans treated with an MRI-linac. These irradiator/magnet combinations are therefore suitable for preclinical studies investigating potential biological effects of delivering radiation therapy in the presence of a magnetic field.

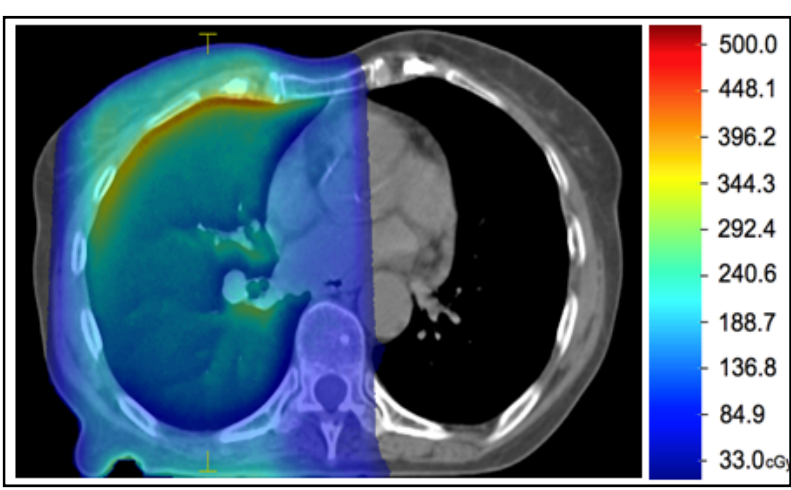

FIG. 1: Human lung irradiation with a single 6MV beam in the presence of a $1.5 \mathrm{~T}$ magnetic field using the Monaco treatment planning system. Hot spots and cold spots are seen at tissue boundaries.

Innovation/impact: Before the recently developed 1.5T/6MV MRI-linac can be used clinically, its safety must be assessed. Magnetic fields are known to alter dose deposition by inducing a reduced build-up dis- 
tance and a laterally shifted, asymmetrical penumbra as well as an effect termed the electron return effect (ERE). Monte Carlo simulations have shown the ERE leads to an increase in dose at the transition from high-density to low-density areas and a decrease in dose when going in the opposite direction. Magnetic field-induced hot spots and cold spots could have potential radiobiological effects. Preclinical experiments should therefore be performed before patients are treated with the MRI-linac. In this study, we determined multiple beam energy/magnetic field combinations that produce dose distributions in the lungs of mice similar to those seen in simulated human treatments with the MRI-linac. These irradiator/magnet combinations can now be used in experiments to investigate magnetic field dose effects in vivo.

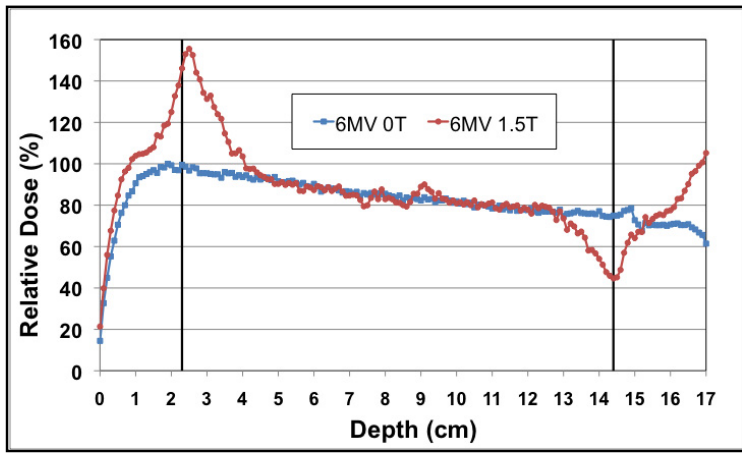

FIG. 2: Percent depth dose (PDD) curves along the central axis of the beam in FIG. 1 in the presence and absence of a magnetic field. Doses are relative to $\mathrm{D}_{\max }$ for the $0 \mathrm{~T}$ case.

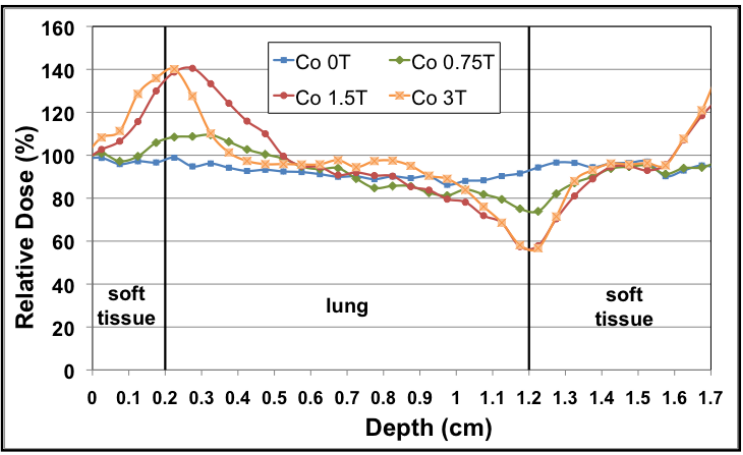

FIG. 3: PDD curves for a $1.25 \mathrm{MeV}$ Co- 60 beam with various magnetic fields in a mouse-sized lung phantom. Material was added anteriorly to allow for $5 \mathrm{~mm}$ of buildup.

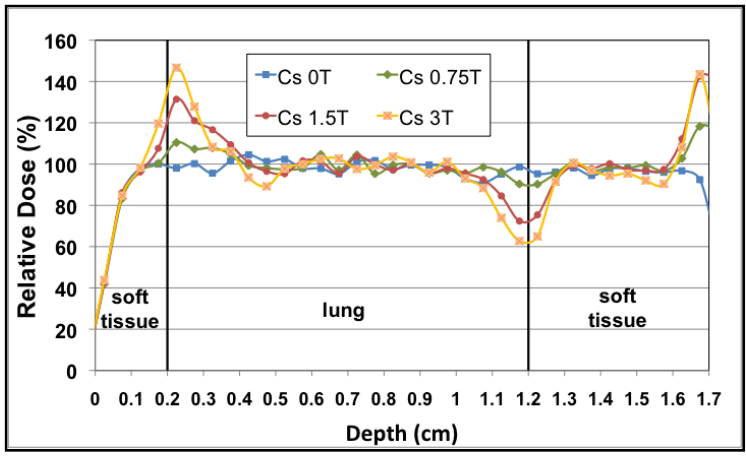

FIG. 4: PDD curves for a $662 \mathrm{keV} \mathrm{Cs-137} \mathrm{beam} \mathrm{with} \mathrm{various} \mathrm{mag-}$ netic fields in a mouse-sized lung phantom. 